\title{
A Finite-time Convergent Observer with Robustness to Piecewise-constant Measurement Noise ${ }^{\star}$
}

\author{
Yuchun Li ${ }^{\text {a }}$, Ricardo G. Sanfelice ${ }^{\mathrm{a}}$ \\ ${ }^{a}$ Department of Computer Engineering, University of California, 1156 High Street, Santa Cruz, CA 95064, USA.
}

\begin{abstract}
Motivated by the design of observers with good performance and robustness to measurement noise, the problem of estimating the state of a linear time-invariant system in finite time and robustly with respect to measurement noise is considered. Using a hybrid systems framework, a hybrid observer producing an estimate that converges to the plant state in finite time, even under unknown piecewise-constant noise, is presented. The stability and robustness properties of the observer are shown analytically and validated numerically.
\end{abstract}

Key words: Estimation; Observer; Robustness.

\section{Introduction}

For a linear time-invariant system defined as

$$
\dot{x}=A x, \quad y=H x+m,
$$

where $x \in \mathbb{R}^{n}, y \in \mathbb{R}^{p}$, and $m: \mathbb{R}_{>0} \rightarrow \mathbb{R}^{p}$ denotes measurement noise, a Luenberger observer is given by

$$
\dot{\hat{x}}_{0}=A \hat{x}_{0}-L_{0}\left(\hat{y}_{0}-y\right), \quad \hat{y}_{0}=H \hat{x}_{0} .
$$

It leads to the estimation error $e_{0}:=\hat{x}_{0}-x$ with dynamics

$$
\dot{e}_{0}=\left(A-L_{0} H\right) e_{0}+L_{0} m .
$$

The matrices $A, H$, and $L_{0}$ are of appropriate dimensions. When the plant (1) is observable, the gain $L_{0}$ can be chosen such that the rate of convergence of $(3)$ is arbitrarily fast; however, due to the term $L_{0} m$ in (3), large gain amplifies the effect of measurement noise. In fact, the design of observers in form (2) involves a tradeoff between the rate of convergence and robustness to measurement noise [1,2]. For different observer structures, researchers have proposed ways to balance this tradeoff. In many applications, using two sets of gains for the observer, one optimized for rate of convergence

\footnotetext{
* This paper was not presented at any IFAC meeting. Corresponding author Y. Li. Tel. 001 831-459-1016.

Email addresses: yuchunli@ucsc.edu (Yuchun Li), ricardo@ucsc.edu (Ricardo G. Sanfelice).
}

and the other one for robustness, works well. Recent results following such an approach involve the hybrid approach in [3], consisting of resetting the gain according to the plant's output norm, the piecewise-linear gain method in [4], which compensates the steady-state and bounds on transient behavior simultaneously, the nonlinear adaptive high-gain observer in [5], and the online gain scheduling high-gain observer in [6]. In [7], a distributed state observer is proposed to relax the said tradeoff.

For scenarios where fast rate of convergence is of main importance, several observer architectures that guarantee finite time convergence of the estimates without measurement noise are available in the literature. These include those using the properties of the solutions of multiple observers, see, e.g., [8-11], using measurement-based state updates [12], and those exploiting an homogeneity property, see, e.g., [13-16]. When noise is present in the measurements of the state of the plant, a tradeoff between rate of convergence and robustness to measurement noise is also expected in finite-time convergent observers. In fact, consider the finite-time convergent observer proposed in [8] without noise, i.e., $m \equiv 0$ and $y=H x$, which is defined as ${ }^{1}$

$$
\begin{aligned}
& \dot{\hat{x}}_{i}(t)=A \hat{x}_{i}(t)-L_{i}\left(H \hat{x}_{i}(t)-y(t)\right) \quad \forall t \neq k \delta, k \in \mathbb{N}, \\
& \hat{x}_{i}^{+}=\tilde{K}_{1, i}(k) \hat{x}_{1}(t)+\tilde{K}_{2, i}(k) \hat{x}_{2}(t) \quad \forall t=k \delta, k \in \mathbb{N},
\end{aligned}
$$

\footnotetext{
$\overline{1 \hat{x}_{i}^{+}(t)}:=\lim _{t^{\prime} \searrow t} \hat{x}_{i}\left(t^{\prime}\right)$, where $t \in[0, \infty)$ is a time instant at which a jump occurs. Equivalently, at times, we write $\hat{x}_{i}^{+}$.
} 
for each $i \in\{1,2\}$, where $\mathbb{N}:=\{1,2,3, \ldots\} ; \hat{x}_{1}, \hat{x}_{2} \in \mathbb{R}^{n}$; $\delta>0 ; F_{1}=A-L_{1} H, F_{2}=A-L_{2} H$, and $L_{1}, L_{2} \in \mathbb{R}^{n \times p} ;$ $\tilde{K}_{2, i}(1)=\left(I-\exp \left(F_{2} \delta\right) \exp \left(-F_{1} \delta\right)\right)^{-1}$ and $\tilde{K}_{1, i}(1)=$ $I-\tilde{K}_{2, i}(1) ; \tilde{K}_{1,1}(k)=I, \tilde{K}_{2,1}(k)=0$ for each $k \in$ $\{2,3,4, \ldots\} ; \tilde{K}_{1,2}(k)=0, \tilde{K}_{2,2}(k)=I$ for each $k \in$ $\{2,3,4, \ldots\}$; see $[8,9]$ for more details. The parameter $\delta$ defines the time that $e_{i}^{+}(\delta)=0$ for each $i \in\{1,2\}$, where the $e_{i}$ 's define the estimation error, i.e., $e_{i}:=\hat{x}_{i}-x$. Based on [8,9], finite-time convergence occurs (at $t=\delta$ ) when $\hat{x}_{1}(0)=\hat{x}_{2}(0)$ if $\tilde{K}_{1, i}(1)$ and $\tilde{K}_{2, i}(1)$ are well defined, which is guaranteed when parameters $L_{1}, L_{2}$, and $\delta$ are chosen to satisfy the following conditions:

\section{Assumption 1.1}

The parameters $L_{1}, L_{2} \in \mathbb{R}^{n \times p}$ and $\delta>0$ are such that

(A1) $F_{i}=A-L_{i} H$ is Hurwitz for each $i \in\{1,2\}$;

(A2) $I-\exp \left(F_{2} \delta\right) \exp \left(-F_{1} \delta\right)$ is invertible.

Figure 1(a) illustrates the tradeoff between rate of convergence and robustness to measurement noise when (4) is used for the scalar plant $\dot{x}=a x, y=x+m$. For constant noise $m$, the $x$-axis of the plot denotes the time when the estimate is reset and the $y$-axis denotes the corresponding error after the reset; in particular, it shows $\left|e_{1}^{+}\right|$at $t=\delta$. Note that $e_{2}^{+}=e_{1}^{+}$. It can be seen that the sooner the observer jumps ( $\delta$ small $)$, the larger the effect of measurement noise after the reset would be. This trend can be justified analytically by studying (4) with measurement noise. Because of the presence of noise, at the reset time $\delta$, the estimate $\hat{x}_{1}, \hat{x}_{2}$ initialized at $\hat{x}_{1}(0)=\hat{x}_{2}(0)$ will not be mapped to $x$. Instead, the error after the jump is given by

$$
\begin{aligned}
e_{i}^{+}(\delta)= & \frac{m}{\exp \left(\tilde{a}_{2} \delta\right)-\exp \left(\tilde{a}_{1} \delta\right)}\left(\frac{L_{1}}{\tilde{a}_{1}} \exp \left(\tilde{a}_{2} \delta\right)\left(\exp \left(\tilde{a}_{1} \delta\right)-1\right)\right. \\
& \left.-\frac{L_{2}}{\tilde{a}_{2}} \exp \left(\tilde{a}_{1} \delta\right)\left(\exp \left(\tilde{a}_{2} \delta\right)-1\right)\right)
\end{aligned}
$$

where $\tilde{a}_{i}=a-L_{i}$ and $m$ is considered constant for the time being. Let $a=-0.05, L_{1}=0.01$ and $L_{2}=0.02$. The evaluation of (5) leads to the plot in Figure 1(a). Figure 1(b) illustrates the said tradeoff by comparing a trajectory of (4) with the one of the Luenberger observer in (2) with gain $L_{0}=0.02$. The red dot line denotes the evolution of the scalar plant $(x \equiv 0)$, the black dash-dot line denotes the trajectory of the Luenberger observer, while the blue solid line denotes the estimation from observer (4), namely, $\hat{x}_{2}\left(\hat{x}_{1}\right.$ has similar behavior $)$ with $\delta=2$. Due to the jump, the estimation from observer (4) approaches zero much faster than that of the Luenberger observer, however, convergence of the estimate does not occur in finite time. ${ }^{2}$

\footnotetext{
2 The effect of measurement noise pointed out above is generic for the scalar system under analysis. In fact, for a general bounded (measurable) noise signal $t \mapsto m(t), e_{i}^{+}(\delta)$ can be upper bounded by $e_{1}^{+}(\delta)=$
}

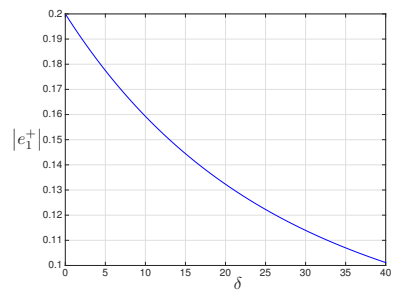

(a) Tradeoff between the rate of convergence and the effect of measurement noise (constant) for a scalar plant.

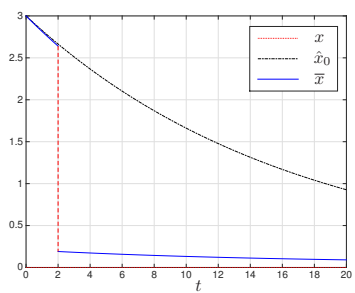
finite-time convergent observer $(\bar{x})$ compared to a Luenberger observer $\left(\hat{x}_{0}\right)$ for the zero solution to (1). (b) Performance of the

Fig. 1. Effect of noise $m$ on the finite-time convergent observer in (4).

To overcome the negative effect of measurement noise in the finite-time convergent observer (4), building from the construction in [17, Example 7.6], we propose a hybrid observer that, for constant or piecewise-constant noise $m$, estimates the state of the plant in finite time with zero error. Under the presence of generic measurement noise and a class of unmodeled dynamics, the proposed observer also induces a $\mathcal{K} \mathcal{L}$-like estimate in the estimation error. In particular, it compensates for the effect of the piecewise-constant bias portion of the generic noise. The strategy proposed here combines the estimates produced by a pair of Luenberger observers, which, when no noise is present, induces a $\mathcal{K} \mathcal{L}$ bound relative to the set of points where the plant and estimates coincide. Moreover, to handle the noise, an additional variable is used to exactly estimate the measurement noise after a finite number of jumps. By estimating the noise at jumps, a final estimate converging to the plant's state after finite jumps is generated. While estimation of constant noise can be performed with tools already available in the literature, the new hybrid observer not only induces an asymptotic stability property that is robust (to general noise and to a class of unmodeled dynamics), but also, when the noise is piecewise-constant, the noise estimate converges to the actual noise after no more than three consecutive jumps, allowing for perfect estimation of the plant's state in finite time. Note that many bias estimation techniques in the literature involve sign functions (see, e.g., [18]), which makes the establishment of robustness somewhat difficult.

$e_{2}^{+}(\delta) \leq \Gamma(\delta)$, where the function $\delta \mapsto \Gamma(\delta)$ is defined as $\Gamma(\delta)=\left|\frac{\exp \left(\tilde{a}_{2} \delta\right)}{\exp \left(\tilde{a}_{2} \delta\right)-\exp \left(\tilde{a}_{1} \delta\right)}\right| \frac{\left|L_{1}\right|}{\left|\tilde{a}_{1}\right|} \sup _{t \in[0, \delta]}|m(t)|+$ $\left|\frac{\exp \left(\tilde{a}_{1} \delta\right)}{\exp \left(\tilde{a}_{2} \delta\right)-\exp \left(\tilde{a}_{1} \delta\right)}\right| \frac{\left|L_{2}\right|}{\left|\tilde{a}_{2}\right|} \sup _{t \in[0, \delta]}|m(t)|$. Without loss of generality, let $L_{2}>L_{1}>0$. Then, it follows that $\lim _{\delta \rightarrow 0^{+}} \Gamma(\delta)=$ $\infty$ and $\lim _{\delta \rightarrow \infty} \Gamma(\delta)=\frac{\left|L_{2}\right|}{\left|\tilde{a}_{2}\right|}|m|_{\infty}$. Moreover, it can be shown that $\frac{d \Gamma}{d \delta}(\delta)<0$ for all $\delta \geq 0$. Therefore, the function $\Gamma$ is strictly decreasing. The upper bound (though it is conservative) implies the same tradeoff between the error after reset and the reset time. More results about the effect of noise to (4) are presented in Appendix B. 
The remainder of this paper is organized as follows. Section 2 introduces notations and the hybrid systems framework employed in this work. Section 3 presents the proposed hybrid observer. In that section, its stability and finite-time convergence properties are established. Furthermore, also in Section 3, the robustness of the proposed hybrid observer to piecewise-constant and general noises as well as a class of unmodeled dynamics are given in terms of $\mathcal{K} \mathcal{L}$-like bounds. Section 4 presents numerical results validating and highlighting the robustness properties of the proposed observer.

\section{Preliminaries}

\subsection{Notation}

$\mathbb{N}_{0}$ denotes the set of natural numbers including zero, namely, $\mathbb{N}_{0}:=\{0,1,2,3, \ldots\} . \mathbb{R}_{>0}$ denotes $[0, \infty)$. Given vectors $\nu \in \mathbb{R}^{n}$ and $w \in \mathbb{R}^{m},|\nu|$ defines the Euclidean vector norm $|\nu|=\sqrt{\nu^{\top} \nu}$, and $\left[\begin{array}{ll}\nu^{\top} & w^{\top}\end{array}\right]^{\top}$ is equivalent to $(\nu, w)$. Given a matrix $A \in \mathbb{R}^{n \times n}$, eig $(A)$ denotes the set of all eigenvalues of $A ; \bar{\mu}(A):=\max \left\{\lambda / 2: \lambda \in \operatorname{eig}\left(A+A^{\top}\right)\right\}$; $\underline{\mu}(A):=\min \left\{\lambda / 2: \lambda \in \operatorname{eig}\left(A+A^{\top}\right)\right\} ; A$ is called dissipative if $\bar{\mu}(A)<0 ;|A|:=\max \left\{|\lambda|^{\frac{1}{2}}: \lambda \in \operatorname{eig}\left(A^{\top} A\right)\right\}$; $\kappa(A):=\min \left\{|X|\left|X^{-1}\right|: A=X J X^{-1}\right\}$ where $J$ is the Jordan normal form of $A ; \bar{\alpha}(A):=\max \{\operatorname{Re}(\lambda)$ : $\lambda \in \operatorname{eig}(A)\} ; \underline{\alpha}(A):=\min \{\operatorname{Re}(\lambda): \lambda \in \operatorname{eig}(A)\}$; with $p \in \mathbb{N}, \operatorname{diag}_{p}(A)$ defines a diagonal square matrix which has $p$ sub-matrices $A$ on the diagonal. The set $\mathbb{B}$ denotes the open unit ball centered at the origin in a Euclidean space. Given a function $f: \mathbb{R}^{n} \rightarrow \mathbb{R}^{p}$, its domain of definition is denoted by $\operatorname{dom} f$, i.e., dom $f:=\left\{x \in \mathbb{R}^{n}: f(x)\right.$ is defined $\}$. Given a function $\mathbb{R}_{\geq 0} \ni t \mapsto m(t) \in \mathbb{R}^{n},|m|_{\infty}:=\sup _{t \in \operatorname{dom} m}|m(t)|$ and $|m|_{\delta}:=\sup _{t \in[0, \delta] \cap \operatorname{dom} m}|m(t)|$. Given a set $B \subset \mathbb{R}^{n}$, the indicator function $\chi_{B}: \mathbb{R}^{n} \rightarrow\{0,1\}$ is defined as $\chi_{B}(x):=0$ if $x \notin B$, and $\chi_{B}(x):=1$ if $x \in B$. A function $\beta: \mathbb{R}_{\geq 0} \times \mathbb{R}_{\geq 0} \rightarrow \mathbb{R}_{\geq 0}$ is a class- $\mathcal{K} \mathcal{L}$ function if it is nondecreasing in its first argument, nonincreasing in its second argument, $\lim _{r \searrow 0} \beta(r, s)=0$ for each $s \in \mathbb{R}_{\geq 0}$, and $\lim _{s \rightarrow \infty} \beta(r, s)=0$ for each $r \in \mathbb{R}_{\geq 0}$. Given a point $y \in \mathbb{R}^{n}$ and a closed set $\mathcal{A} \subset \mathbb{R}^{n},|y|_{\mathcal{A}}:=\inf _{x \in \mathcal{A}}|x-y|$. The set $\left\{v_{i}\right\}_{i \in\{1,2, \ldots, n\}}$ defines an orthonormal basis for $\mathbb{R}^{n}$, where each column vector $v_{i}$ contains the only nonzero element 1 at the $i$-th entry. Given matrices $A$ and $B$ with proper dimensions, $\operatorname{He}(A, B):=A^{\top} B+B A$.

\subsection{Preliminaries on hybrid systems}

In this paper, a hybrid system $\mathcal{H}$ has data $(C, f, D, g)$ and is defined by

$$
\begin{aligned}
\dot{z} & =f(z, u) \quad z \in C, \\
z^{+} & =g(z, u) \quad z \in D,
\end{aligned}
$$

where $z \in \mathbb{R}^{\bar{n}}$ is the state, $u \in \mathbb{R}^{\bar{p}}$ is the input, $f$ defines the flow map which captures the continuous dynamics and $C$ defines the flow set on which $f$ is effective. The map $g$ defines the jump map and models the discrete behavior, while $D$ defines the jump set, from which discrete dynamics are allowed. Given an input $u$, a solution to $\mathcal{H}$ is given by the pair $(z, u)$, which is parametrized by $(t, j)$, where $t$ denotes ordinary time and $j$ denotes the jump time. (When the system has no input or its input is zero, its solution will be given by z.) Hybrid time domains are subsets $E$ of $\mathbb{R}_{>0} \times \mathbb{N}_{0}$ that, for each $(T, J) \in E, E \cap([0, T] \times\{0,1, \ldots, J\})$ can be written in the form $\cup_{j=0}^{J-1}\left(I_{j}, j\right)$ for some finite sequence of times $0=t_{0} \leq t_{1} \leq t_{2} \leq \cdots \leq t_{J}$, where $I_{j}:=\left[t_{j}, t_{j+1}\right]$. The $t_{j}$ 's define the time instants when the state of the hybrid system jumps and $j$ counts the time of jumps. A solution to $\mathcal{H}$ is called maximal if it cannot be extended, i.e., it is not a truncated version of another solution, and it is called complete if its domain is unbounded. A solution is called Zeno if it is complete and its domain is bounded in the $t$ direction. Two solutions are said to be $\left(\tau^{\prime}, \varepsilon\right)$-close if they satisfy the following property.

Definition 2.1 ( $\left(\tau^{\prime}, \varepsilon\right)$-closeness of solutions [17, Definition 5.23]) Given $\tau^{\prime}, \varepsilon>0$, two solutions $\phi_{1}$ and $\phi_{2}$ to $\mathcal{H}$ are $\left(\tau^{\prime}, \varepsilon\right)$-close if

(a) for all $(t, j) \in \operatorname{dom} \phi_{1}$ with $t+j \leq \tau^{\prime}$ there exists $s$ such that $(s, j) \in \operatorname{dom} \phi_{2},|t-s|<\varepsilon$, and $\mid \phi_{1}(t, j)-$ $\phi_{2}(s, j) \mid<\varepsilon$

(b) for all $(t, j) \in \operatorname{dom} \phi_{2}$ with $t+j \leq \tau^{\prime}$ there exists $s$ such that $(s, j) \in \operatorname{dom} \phi_{1},|t-s|<\varepsilon$, and $\mid \phi_{2}(t, j)-$ $\phi_{1}(s, j) \mid<\varepsilon$.

A hybrid system $\mathcal{H}$ with $(C, f, D, g)$ is said to satisfy the hybrid basic conditions [17, Assumption 6.5] if the following hold:

(B1) $C$ and $D$ are closed sets in $\mathbb{R}^{\bar{n}}$;

(B2) the functions $f$ and $g$ are continuous.

The hybrid observer proposed in the next section has $y$ as its input, while for the analysis of robustness to noise, an exogenous signal $m$ defining measurement noise will play the role of $u$. A mapping $m$ is admissible if dom $m$ is a hybrid time domain and, for each $j \in \mathbb{N}_{0}$, the function $t \rightarrow m(t, j)$ is measurable. We refer the reader to $[17,19]$ for more details on this hybrid systems framework.

\section{Robust Finite-time Convergent Hybrid Ob- server}

This section presents the proposed hybrid observer and its main properties. The proposed observer, denoted $\mathcal{H}_{o}$ and with data $\left(C_{o}, f_{o}, D_{o}, g_{o}\right)$, has state $\zeta_{o}=\left(\hat{x}_{1}, \hat{x}_{2}, \xi_{1}, \xi_{2}, \hat{m}, \tau\right) \in \mathcal{X}_{o}:=\mathbb{R}^{n} \times \mathbb{R}^{n} \times \mathbb{R}^{n p} \times$ 
$\mathbb{R}^{n p} \times \mathbb{R}^{p} \times[0, \delta]$ and input $y \in \mathbb{R}^{p}$. The flow map and jump map are given by ${ }^{3}$

$$
\begin{aligned}
& f_{o}\left(\zeta_{o}, y\right)=\left[\begin{array}{l}
A \hat{x}_{1}+L_{1}\left(y-H \hat{x}_{1}\right)-L_{1} \hat{m} \\
A \hat{x}_{2}+L_{2}\left(y-H \hat{x}_{2}\right)-L_{2} \hat{m} \\
\operatorname{diag}_{p}\left(F_{1}\right) \xi_{1}+\tilde{L}_{1} \\
\operatorname{diag}_{p}\left(F_{2}\right) \xi_{2}+\tilde{L}_{2} \\
0 \\
1
\end{array}\right], \\
& g_{o}\left(\zeta_{o}, y\right)=\left[\begin{array}{l}
R-T \psi(H R-(y-\hat{m})) \\
R-T \psi(H R-(y-\hat{m})) \\
0 \\
0 \\
\hat{m}+\psi(H R-(y-\hat{m})) \\
0
\end{array}\right],
\end{aligned}
$$

respectively, where, for each $i \in\{1,2\}$,

$$
\begin{aligned}
& R\left(\hat{x}_{1}, \hat{x}_{2}\right)=K_{1} \hat{x}_{1}+K_{2} \hat{x}_{2}, \quad \tilde{L}_{i}=\sum_{k=1}^{p} \mathcal{I}_{k}^{\top} L_{i} v_{k}, \\
& \psi\left(\xi_{1}, \xi_{2}\right)=\left(H T\left(\xi_{1}, \xi_{2}\right)-I\right)^{-1}, \quad F_{i}=A-L_{i} H, \\
& T\left(\xi_{1}, \xi_{2}\right)=K_{1} \sum_{k=1}^{p} \mathcal{I}_{k} \xi_{1} v_{k}^{\top}+K_{2} \sum_{k=1}^{p} \mathcal{I}_{k} \xi_{2} v_{k}^{\top}, \\
& K_{1}=I-K_{2}, \quad K_{2}=\left(I-\exp \left(F_{2} \delta\right) \exp \left(-F_{1} \delta\right)\right)^{-1}
\end{aligned}
$$

with $\mathcal{I}_{k} \in \mathbb{R}^{n \times n p}$ defined by $p$ matrices of dimension $n \times n$ in a row, of which the only nonzero sub-matrix among them is the identity matrix $I_{n \times n}$ located at the $k$-th entry, e.g., $\mathcal{I}_{k}=\left[\begin{array}{llll}0_{n \times n} & 0_{n \times n} \ldots 0_{n \times n} & I_{n \times n} & 0_{n \times n}\end{array}\right]$. The flow set is defined by $C_{o}:=\mathcal{X}_{o}$ and the jump set is given by $D_{o}:=\left\{\zeta_{o} \in \mathcal{X}_{o}: \tau=\delta\right\}$. These definitions of $C_{o}$ and $D_{o}$ ensure that (possibly after the first jump) the system jumps periodically.

The hybrid observer $\mathcal{H}_{o}$ interconnected with the plant (1) defines a hybrid system $\mathcal{H}=(C, f, D, g)$ with state $z=\left(x, \zeta_{o}\right)$, input $m, C=\mathbb{R}^{n} \times C_{o}$, $f(z, m)=\left(A x, f_{o}\left(\zeta_{o}, y\right)\right), D=\mathbb{R}^{n} \times D_{o}$, and $g(z, m)=$ $\left(x, g_{o}\left(\zeta_{o}, y\right)\right)$. For this interconnection, we have the following nominal property.

Theorem 3.1 For the interconnection between $\mathcal{H}_{o}$ and the plant (1) with $z:=\left(x, \zeta_{o}\right)$, suppose Assumption 1.1 holds and the measurement noise $m$ is zero. Moreover, suppose $\Upsilon:=H \sum_{i=1}^{2} K_{i} \int_{0}^{\delta} \exp \left(F_{i} \tau\right) L_{i} d \tau-I$ is invertible. Let $\mathcal{A}=\left\{z \in \mathbb{R}^{n} \times \mathcal{X}_{o}: x=\hat{x}_{1}=\hat{x}_{2}\right\}$. Then, each

3 For simplicity, the arguments in $\psi, T$, and $R$ are dropped in $(7 \mathrm{a})$ and $(7 \mathrm{~b})$. solution to the interconnection from $z(0,0) \in S:=\{z \in$ $\left.\mathbb{R}^{n} \times \mathcal{X}_{o}: \hat{x}_{1}=\hat{x}_{2}, \xi_{1}=\xi_{2}=0, \tau=0, \hat{m}=0\right\}$ satisfies

$$
|z(t, j)|_{\mathcal{A}} \leq \rho(j) \sqrt{\frac{w_{2}}{w_{1}}}|z(0,0)|_{\mathcal{A}} \exp \left(\frac{\bar{\alpha}(Q)}{2 w_{2}} t\right)
$$

for all $(t, j) \in \operatorname{dom} z$ with $\rho(0)=1$ and $\rho(j)=0$ for each $j \in \mathbb{N}, w_{1}=\min \left\{\underline{\alpha}\left(P_{1}\right), \underline{\alpha}\left(P_{2}\right)\right\}, w_{2}=$ $3 \max \left\{\bar{\alpha}\left(P_{1}\right), \bar{\alpha}\left(P_{2}\right)\right\}$, for any $P_{1}=P_{1}^{\top}>0$ and $P_{2}=P_{2}^{\top}>0$ such that

$$
Q:=\left[\begin{array}{cc}
\operatorname{He}\left(F_{1}, P_{1}\right) & 0 \\
0 & \operatorname{He}\left(F_{2}, P_{2}\right)
\end{array}\right]<0 .
$$

Proof To show the $\mathcal{K} \mathcal{L}$-like bound in (8), consider a Lyapunov candidate $V(z)=\sum_{i=1}^{2}\left(x-\hat{x}_{i}\right)^{\top} P_{i}\left(x-\hat{x}_{i}\right)$, where, for each $i \in\{1,2\}, P_{i} \in \mathbb{R}^{n \times n}$ is a symmetric positive definite matrix. Then, it satisfies the property

$$
w_{1}|z|_{\mathcal{A}}^{2} \leq V(z) \leq w_{2}|z|_{\mathcal{A}}^{2} \quad \forall z \in C \cup D \cup g(D),
$$

where $w_{1}=\min \left\{\underline{\alpha}\left(P_{1}\right), \underline{\alpha}\left(P_{2}\right)\right\}$ and $w_{2}=3 \max \left\{\bar{\alpha}\left(P_{1}\right), \bar{\alpha}\left(P_{2}\right)\right\}$.

For each $z \in C$ and $\hat{m}=0$ :

$$
\langle\nabla V(z), f(z)\rangle=\left[\left(x-\hat{x}_{1}\right)^{T}\left(x-\hat{x}_{2}\right)^{T}\right] Q\left[\begin{array}{l}
x-\hat{x}_{1} \\
x-\hat{x}_{2}
\end{array}\right],
$$

with $Q$ defined in (9). The time derivative of the Lyapunov function is negative definite since, by condition (A1) in Assumption 1.1, the matrix $Q$ is negative definite. Then, we have

$\langle\nabla V(z), f(z)\rangle \leq \bar{\alpha}(Q)|z|_{\mathcal{A}}^{2} \quad \forall z \in C$ and $\hat{m}=0$.

Now, consider the change of $V$ at jumps. For each maximal solution $z$ to the interconnection from $z(0,0) \in S$, and each $(t, j) \in \operatorname{dom} z$ such that $(t, j+1) \in \operatorname{dom} z$, by using the definitions of $K_{1}$ and $K_{2}$, we obtain that

$V(g(z(t, j)))-V(z(t, j))=-V(z(t, j)) \leq-w_{1}|z(t, j)|_{\mathcal{A}}^{2}$

and that $g(z(t, j)) \in S$. Then, by direct integration and using bounds (10) and (11), for any solution of the system initialized at $z(0,0) \in S$, we have (8).

Remark 3.2 The explicit $\mathcal{K} \mathcal{L}$ bound in (8) provides a useful estimate on the overshoot during transient. Moreover, the interconnection between $\mathcal{H}_{o}$ and the plant (1) is such that every maximal solution is complete and the 
invertibility property of $\Upsilon$ can be checked offline ${ }^{4}$ (similarly, $T\left(\xi_{1}, \xi_{2}\right)$ can be evaluated offline). Furthermore, when $z(0,0)$ is not restricted to $S$, i.e., $z(0,0) \in \mathbb{R}^{n} \times \mathcal{X}_{o}$, the bound in (8) can be extended to capture the transient due to initial conditions not in $S$, in which case, to avoid singularity, one could replace $\psi$ by the set-valued map

$$
\psi\left(\xi_{1}, \xi_{2}\right)= \begin{cases}\Delta\left(\xi_{1}, \xi_{2}\right)^{-1} & \text { if }\left|\operatorname{det}\left(\Delta\left(\xi_{1}, \xi_{2}\right)\right)\right|>\Lambda \\ 0 & \text { if }\left|\operatorname{det}\left(\Delta\left(\xi_{1}, \xi_{2}\right)\right)\right|<\Lambda \\ \left\{0, \Delta\left(\xi_{1}, \xi_{2}\right)^{-1}\right\} & \text { if }\left|\operatorname{det}\left(\Delta\left(\xi_{1}, \xi_{2}\right)\right)\right|=\Lambda\end{cases}
$$

for each $\xi_{1}, \xi_{2} \in \mathbb{R}^{n p}$, where $\Delta\left(\xi_{1}, \xi_{2}\right)=H T\left(\xi_{1}, \xi_{2}\right)-I$ and $\Lambda>0$ is a small parameter such that

$$
\left|\operatorname{det}\left(H \sum_{i=1}^{2} K_{i} \int_{0}^{\delta} \exp \left(F_{i} \tau\right) L_{i} d \tau-I\right)\right|>\Lambda .
$$

The following result establishes the main convergence property induced by $\mathcal{H}_{o}$ when piecewise-constant measurement noise is present.

Theorem 3.3 For the interconnection between the observer $\mathcal{H}_{o}$ and the plant (1) with $z=\left(x, \zeta_{o}\right)$, assume the measurement noise $m$ is a piecewise-constant function defined as

$$
m(t):=\sum_{k} \tilde{c}_{k} \chi_{B_{k}}(t) \quad \forall t \in[0, \infty)
$$

where, for integers $k \geq 1, \tilde{c}_{k} \in \mathbb{R}^{p}, B_{k}:=\left[\tilde{d}_{k-1}, \tilde{d}_{k}\right)$ with $0 \leq \tilde{d}_{k-1}<\tilde{d}_{k}, \tilde{\tilde{d}}_{k}$ finite or infinite, and $\cup_{k} B_{k}=$ $[0, \infty)$. Moreover, suppose Assumption 1.1 holds, $0<\delta<$ $\frac{1}{2} \min _{k \in \mathbb{N}}\left\{\tilde{d}_{k}-\tilde{d}_{k-1}\right\}$, and $\Upsilon$ is invertible. Then, each solution $z$ to the interconnection with $z(0,0) \in S^{\star}:=$ $\left\{z \in \mathbb{R}^{n} \times \mathcal{X}_{o}: \xi_{1}=\xi_{2}=0, \tau=0\right\}$, is such that, for each $j \geq 1, I_{j} \times\{j\} \in \operatorname{dom} z$, there exists $\tilde{I}_{j} \subset\left(I_{j} \cup I_{j+1}\right)$ with nonempty interior such that $\hat{x}_{i}(t, j)=x(t, j)$ for each $t \in \tilde{I}_{j}$ and each $i \in\{1,2\}$.

Proof Consider the first two intervals $I_{0}, I_{1}$ of a solution $z$ to the interconnection from $z(0,0) \in S^{\star}$. Under the assumption that $0<\delta<\frac{1}{2} \min _{k \in \mathbb{N}}\left\{\tilde{d}_{k}-\tilde{d}_{k-1}\right\}$, the measurement noise $m$ is constant for all $t \in I_{0} \cup I_{1}$ (note we can rewrite $m$ in (13) as a function on dom $z$; see $[20])$. Applying the jump map (7b), we obtain $\hat{x}_{1}(\delta, 1)=$ $\hat{x}_{2}(\delta, 1)$ after the first jump. Recall that the measurement

\footnotetext{
4 For a scalar plant $(H=1), \Upsilon$ reduces to $\nu_{1} \frac{1-\exp \left(F_{1} \delta\right)}{\left(1-\exp \left(-\left(L_{1}-L_{2}\right) \delta\right)\right)}+\nu_{2} \frac{1-\exp \left(F_{2} \delta\right)}{1-\exp \left(\left(L_{1}-L_{2}\right) \delta\right)}-1$ with $\nu_{1}=$ $-\frac{L_{1}}{F_{1}}$ and $\nu_{2}=-\frac{L_{2}}{F_{2}}$. Let $\Sigma(\delta)=\nu_{1} \frac{1-\exp \left(F_{1} \delta\right)}{\left(1-\exp \left(-\left(L_{1}-L_{2}\right) \delta\right)\right)}+$ $\nu_{2} \frac{1-\exp \left(F_{2} \delta\right)}{1-\exp \left(\left(L_{1}-L_{2}\right) \delta\right)}-1$ for $\delta \in \mathbb{R}_{\geq 0}$. Then, it can be shown that $\lim _{\delta \rightarrow 0} \Sigma(\delta)=0$ and $\lim _{\delta \rightarrow \infty} \Sigma(\delta)=\nu_{2}-1$, where $0<\nu_{2}<1$ for $a<0$ and $L_{2}>0$. Moreover, $\Sigma: \mathbb{R}_{\geq 0} \rightarrow \mathbb{R}$ is continuous and monotonically decreasing. Therefore, $\Sigma(\delta)$ is invertible for any $\delta>0$.
}

noise $m$ is constant for each $t \in I_{1}$. Applying Lemma A.1, it follows that there exists a nonempty sub-interval of $I_{2}$ such that $\hat{x}_{1}(t, j)=\hat{x}_{2}(t, j)=x(t, j)$ for all $t$ belonging to that sub-interval. For any two general consecutive intervals $I_{j} \cup I_{j+1}$ for $j \geq 2$,

- if $\hat{x}_{1}(j \delta, j)=\hat{x}_{2}(j \delta, j)=x(j \delta, j)$, and the noise $m$ does not change at $t=j \delta$, then, using the assumption that $0<\delta<\frac{1}{2} \min _{k \in \mathbb{N}}\left\{\tilde{d}_{k}-\tilde{d}_{k-1}\right\}$, there exists a nonempty sub-interval of $I_{j}$ such that $m$ is constant on this interval, and $\hat{x}_{1}(t, j)=\hat{x}_{2}(t, j)=x(t, j)$ for all $t$ in that sub-interval;

- if $\hat{x}_{1}(j \delta, j)=\hat{x}_{2}(j \delta, j)=x(j \delta, j)$, and the noise $m$ changes at $t=j \delta$, it will keep constant for $t \in I_{j} \cup I_{j+1}$, then it follows from an application of Lemma A.1 as above that $\hat{x}_{1}(t, j)=\hat{x}_{2}(t, j)=x(t, j)$ for all $t \in I_{j+1}$;

- if $\hat{x}_{1}(j \delta, j)=\hat{x}_{2}(j \delta, j) \neq x(j \delta, j)$, it is implied that the noise $m$ changed once in $I_{j-1}$ and it will keep constant for $t \in I_{j}$. Therefore, there exists a nonempty subinterval of $I_{j+1}$ such that $\hat{x}_{1}(t, j+1)=\hat{x}_{2}(t, j+1)=$ $x(t, j+1)$ for all $t$ that belongs to that sub-interval.

The claim holds by combining the arguments above.

The existence of the interval $\tilde{I}_{j}$ for each $j \geq 1$ guaranteed by Theorem 3.3 implies that whenever the noise changes, the proposed observer estimates the new value of the piecewise-constant noise in finite time (within $3 \delta$ ).

Remark 3.4 A special case of Theorem 3.3, which follows from Lemma $A .1$, is that, if the measurement noise $m$ is constant and $\Upsilon$ is invertible, then, for each initial condition $z(0,0) \in S^{\star}$ for the interconnection between the observer $\mathcal{H}_{o}$ and the plant $(1), \hat{x}_{1}$ and $\hat{x}_{2}$ converge to $x$ within $2 \delta$.

To analyze the robustness property to generic measurement noise $m$ of the interconnection between the observer $\mathcal{H}_{o}$ and the plant (1), given $m$ and $\hat{m}$ with $\operatorname{dom} m=\operatorname{dom} \hat{m}$, define $|m-\hat{m}|_{(t, j)}:=$ $\sup _{s \in[j \delta, t]}|m(s, j)-\hat{m}(j \delta, j)|$, for each $(t, j) \in \operatorname{dom} m$ (since $\hat{m}$ does not change over flows, $\hat{m}(s, j)=\hat{m}(j \delta, j)$ for each $s \in[j \delta, t])$ and $|m-\hat{m}|_{(t, j)}:=0$ for each $(t, j) \notin \operatorname{dom} m$.

Theorem 3.5 For the interconnection between the observer $\mathcal{H}_{o}$ and the plant (1) with $z=\left(x, \zeta_{o}\right)$ and an admissible measurement noise $m$, suppose $\bar{\alpha}\left(F_{2}\right)<\underline{\alpha}\left(F_{1}\right)$ and $\bar{\alpha}\left(F_{1}\right)<0$. Furthermore, suppose $F_{1}$ and $F_{2}$ are dissipative and $\bar{\mu}\left(F_{2}\right)<\underline{\mu}\left(F_{1}\right)$, and $\Upsilon$ is invertible. Then, each solution $z$ to the interconnection from $z(0,0) \in$ $S^{\star \star}:=\left\{z \in \mathbb{R}^{n} \times \mathcal{X}_{o}: \hat{x}_{1}=\hat{x}_{2}, \xi_{1}=\xi_{2}=0, \tau=0\right\}$ satisfies, for each $i \in\{1,2\}$ and for all $(t, j) \in$ dom $z$,

$$
\begin{aligned}
\left|e_{i}(t, j)\right| \leq & \rho(j) \exp \left(\bar{\mu}\left(F_{i}\right) t\right)\left|e_{i}(0,0)\right| \\
& +\left((1-\rho(j)) c \exp \left(\bar{\mu}\left(F_{i}\right)(t-j \delta)\right)+d_{i}\right) \\
& \times \max \left\{|m-\hat{m}|_{(j \delta, j-1)},|m-\hat{m}|_{(t, j)}\right\},
\end{aligned}
$$


where $e_{i}=\hat{x}_{i}-x, \rho(j)=1$ if $j=0$ and $\rho(j)=0$ for each $j \in \mathbb{N}, d_{i}=\frac{\left|L_{i}\right|}{\left|\bar{\mu}\left(F_{i}\right)\right|}, c=c_{1}+c_{1} c_{2}\left(|H| c_{1}+1\right)$, $c_{1}=\sum_{i=1}^{2}\left|K_{i}\right| \frac{\left|L_{i}\right|}{\left|\bar{\mu}\left(F_{i}\right)\right|}$, and $c_{2}=\left|\Upsilon^{-1}\right|$.

Proof Consider the interval $[0, \delta] \times\{0\}$ over which no jump occurs. By solving for the solution to the interconnection during flows, we obtain

$$
\left|e_{i}(t, 0)\right| \leq \exp \left(\bar{\mu}\left(F_{i}\right) t\right)\left|e_{i}(0,0)\right|+\frac{\left|L_{i}\right|}{\left|\bar{\mu}\left(F_{i}\right)\right|}|m-\hat{m}|_{(t, 0)}
$$

for all $t \in[0, \delta]$, where we use bounds in [21, Section $3.2]$. At the first jump, when $t=\delta$, recalling the jump map defined in $(7 \mathrm{~b})$, we have

$$
\left|R\left(\hat{x}_{1}(\delta, 0), \hat{x}_{2}(\delta, 0)\right)-x(\delta, 1)\right| \leq c_{1}|m-\hat{m}|_{(\delta, 0)},
$$

which follows from the definition of $K_{i}$, the facts that $\hat{x}_{1}(0,0)=\hat{x}_{2}(0,0)$ and $x(\delta, 1)=x(\delta, 0)$, and a straightforward bound on $e_{i}(\delta, 0)$, and also that

$$
\begin{aligned}
& \left|e_{i}(\delta, 1)\right| \leq\left|R\left(\hat{x}_{1}(\delta, 0), \hat{x}_{2}(\delta, 0)\right)-x(\delta, 1)\right| \\
& \quad+\left|T\left(\xi_{1}(\delta, 0), \xi_{2}(\delta, 0)\right)\right|\left|\psi\left(\xi_{1}(\delta, 0), \xi_{2}(\delta, 0)\right)\right| \\
& \quad \times\left(|H|\left|R\left(\hat{x}_{1}(\delta, 0), \hat{x}_{2}(\delta, 0)\right)-x(\delta, 1)\right|+|m-\hat{m}|_{(\delta, 0)}\right) .
\end{aligned}
$$

Note that $\psi\left(\xi_{1}(\delta, 0), \xi_{2}(\delta, 0)\right)=\Upsilon^{-1}$ since $\xi_{1}(0,0)=$ $\xi_{2}(0,0)=0$ and $\tau(0,0)=0$. These bounds lead to $\left|e_{i}(\delta, 1)\right| \leq c|m-\hat{m}(0,0)|_{(\delta, 0)}$. Then, it follows that, for each $t \in[\delta, 2 \delta]$,

$$
\begin{aligned}
\left|e_{i}(t, 1)\right| \leq & \exp \left(\bar{\mu}\left(F_{i}\right)(t-\delta)\right) c|m-\hat{m}|_{(\delta, 0)} \\
& +\frac{\left|L_{i}\right|}{\left|\bar{\mu}\left(F_{i}\right)\right|}|m-\hat{m}|_{(t, 1)} \\
\leq & \left(c \exp \left(\bar{\mu}\left(F_{i}\right)(t-\delta)\right)+\frac{\left|L_{i}\right|}{\left|\bar{\mu}\left(F_{i}\right)\right|}\right) \\
& \times \max \left\{|m-\hat{m}|_{(\delta, 0)},|m-\hat{m}|_{(t, 1)}\right\} .
\end{aligned}
$$

Following the same procedure for $j>1$, the bound on $\left|e_{i}(j \delta, j)\right|$ at the $j$-th jump is independent from the bound on $\left|e_{i}(t, j-1)\right|$ for each $t \in[(j-1) \delta, j \delta]$, and is given by $\left|e_{i}(j \delta, j)\right| \leq c|m-\hat{m}|_{(j \delta, j-1)}$. Over the flow interval $[j \delta,(j+1) \delta] \times\{j\}$, we obtain

$$
\begin{aligned}
\left|e_{i}(t, j)\right| \leq & \exp \left(\bar{\mu}\left(F_{i}\right)(t-j \delta)\right) c|m-\hat{m}|_{(j \delta, j-1)} \\
& +\frac{\left|L_{i}\right|}{\left|\bar{\mu}\left(F_{i}\right)\right|}|m-\hat{m}|_{(t, j)} \\
\leq & \left(c \exp \left(\bar{\mu}\left(F_{i}\right)(t-j \delta)\right)+\frac{\left|L_{i}\right|}{\left|\bar{\mu}\left(F_{i}\right)\right|}\right) \\
& \times \max \left\{|m-\hat{m}|_{(j \delta, j-1)},|m-\hat{m}|_{(t, j)}\right\} .
\end{aligned}
$$

Therefore, we can unify the bounds for any $(t, j) \in$ dom $e_{i}$, to obtain (14).
In addition to the above results for piecewise-constant noise (Theorem 3.3) and to general noise (Theorem 3.5), the fact that the interconnection between the observer $\mathcal{H}_{o}$ and the plant (1) satisfies the hybrid basic conditions, as stated next, enables us to establish a general robustness result over finite horizons.

Proposition 3.6 Suppose A2 in Assumption 1.1 holds and the measurement noise is zero. Moreover, assume $\Upsilon$ is invertible. Then, the interconnection between $\mathcal{H}_{o}$ and the plant satisfies the hybrid basic conditions.

Next, we consider the effect of general measurement noise and unmodeled dynamics on the plant. In such a setting, the plant in (1) becomes

$$
\dot{x}=(A+\Delta A) x, \quad y=(H+\Delta H) x+m .
$$

The proposed observer uses $A$ and $H$, namely, $\Delta A$ and $\Delta H$ are not known to the observer. Due to this, the hybrid observer $\mathcal{H}_{o}$ interconnected with the perturbed plant (15) defines a hybrid system $\widetilde{\mathcal{H}}=(C, \widetilde{f}, D, \widetilde{g})$ with state $\widetilde{z}=\left(x, \zeta_{o}\right)$, input $m$, and flow map and jump map given by

$\widetilde{f}=f+\left(\Delta A x, L_{1} \Delta H x, L_{2} \Delta H x, 0,0,0,0\right)$,

$\widetilde{g}=g+(0, T \psi \Delta H x, T \psi \Delta H x, 0,0,-\psi \Delta H x, 0)$.

The arguments of the functions in (16) and (17) are dropped for simplicity. The following robustness result relates, over a finite horizon, the solutions $\widetilde{z}$ to the system $\widetilde{\mathcal{H}}$, which includes unmodeled dynamics, to the solutions $z$ to $\mathcal{H}$, which is the hybrid system resulting from the interconnection between $\mathcal{H}_{o}$ and the plant (1). Since to establish it we use tools from [17] for autonomous hybrid systems, we assume that the measurement noise is generated by an exosystem. Let $M>0$ and $\Omega \subset \mathbb{R}^{p}$ be compact. Then, we generate the measurement noise from the differential inclusion with constraints

$$
\dot{m} \in M \mathbb{B} \quad m \in \Omega,
$$

where, with some abuse of notation, $m$ is treated as a state vector and solutions to $\widetilde{\mathcal{H}}$ are given by $(\widetilde{z}, \widetilde{m})$; similarly, solutions to $\mathcal{H}$ are given by $(z, m)$. Every possible solution to (18) is bounded and Lipschitz continuous, but not necessarily differentiable, which allows not only for the generation of the piecewise constant noise signals allowed in Theorem 3.3 but also much richer admissible noise signals. Below, given a set $\mathcal{K} \subset \mathbb{R}^{n}$ and $\tau^{\prime} \geq 0, \mathcal{R}_{\tau^{\prime}}^{x}(\mathcal{K})$ denotes the reachable set of $\dot{x}=A x$ from $\mathcal{K}$ up to $t=\tau^{\prime}$ while $\mathcal{R}_{\tau^{\prime}}^{\xi}(0)$ denotes the reachable set of $\dot{\xi}_{1}=\operatorname{diag}_{p}\left(F_{1}\right) \xi_{1}+\tilde{L}_{1}, \dot{\xi}_{2}=\operatorname{diag}_{p}\left(F_{2}\right) \xi_{2}+\tilde{L}_{2}$ from 0 also up to $t=\tau^{\prime}$.

Theorem 3.7 Suppose Assumption 1.1 holds and that $\Upsilon$ is invertible. For each compact sets $\mathcal{K} \subset \mathbb{R}^{n}$ 
and $\mathcal{K}_{\hat{m}} \subset \mathbb{R}^{p}$, each $\varepsilon>0$, and each $\tau^{\prime} \geq 0$ there exists $\varsigma>0$ such that if $\Delta A$ and $\Delta H$ satisfy $\max \{|\Delta A|,|\Delta H|\} \max _{x \in \mathcal{R}_{\tau^{\prime}}^{x}(\mathcal{K})}|x| \leq \frac{\varsigma}{\rho}$ with

$$
\rho=\max \left\{1,\left|L_{1}\right|,\left|L_{2}\right|, \max _{\left(\xi_{1}, \xi_{2}\right) \in \mathcal{R}_{\tau^{\prime}}^{\xi}(0)}\{|T \psi|,|\psi|\}\right\},
$$

then the following property holds: for every solution $(\widetilde{z}, \widetilde{m})$ to $\widetilde{\mathcal{H}}$ with $(\widetilde{z}(0,0), \widetilde{m}(0,0)) \in\left(\mathcal{X}_{\xi} \cap S^{\star}\right) \times \Omega$ there exists a solution $(z, m)$ to $\mathcal{H}$ with $(z(0,0), m(0,0)) \in$ $\left(\mathcal{X}_{\xi} \cap S^{\star}\right) \times \Omega$ such that $(\widetilde{z}, \widetilde{m})$ and $(z, m)$ are $\left(\tau^{\prime}, \varepsilon\right)$ close, where $\mathcal{X}_{\xi}=\mathcal{K} \times \mathcal{K} \times \mathcal{K} \times \mathbb{R}^{n p} \times \mathbb{R}^{n p} \times \mathcal{K}_{\hat{m}} \times[0, \delta]$.

Proof To establish the $\left(\tau^{\prime}, \varepsilon\right)$-close property, we apply [17, Proposition 6.34]. To this end, note that under Assumption 1.1, Proposition 3.6 implies that $\mathcal{H}$ satisfies the hybrid basic conditions listed in (B1)-(B2) of Section 2.2. Denote by $\mathcal{H}_{\mathrm{ex}}$ the interconnection between the plant in (1) and the noise model in (18), in which, at jumps of $\mathcal{H}, m$ is mapped by the identity. This interconnection is autonomous and satisfies [17, Assumption 6.5] (which is the version of the hybrid basic conditions for the case of $f$ and $g$ being set valued). It follows from [17, Theorem 6.30] that $\mathcal{H}_{\text {ex }}$ is well posed; see [17, Definition 6.27 ] for a definition of well posedness.

Now, as we show next, every maximal solution $(z, m)$ to $\mathcal{H}_{\text {ex }}$ is complete, which implies that $\mathcal{H}_{\text {ex }}$ is pre-forward complete [17, Definition 6.12]. First, every maximal solution $m$ to the exosystem (18) is bounded and complete. Moreover, $m$ enters additively and linearly in $f$ and $g$, in which the factors multiplying it are bounded. Since, at most after the first jump, the time between jumps is equal to $\delta$, solutions to $\mathcal{H}$ from $S^{\star}$ do not escape to infinity in finite time when the measurement noise $m$ is bounded. Denote by $T_{C}(z)$ the tangent cone of $C$ at $z$. Then, for each $z(0,0) \in C \backslash D$ and $m(0,0) \in \Omega$, $T_{C}(z(0,0)) \cap f(z(0,0), m(0,0)) \neq \emptyset$ and $g(D, m) \subset C$ for each $m \in \Omega$. Combining the above arguments it follows that only item (a) in [17, Proposition 6.10] holds, which, in turn, implies that every maximal solution to $\mathcal{H}_{\text {ex }}$ is complete.

Then, invoking [17, Proposition 6.34], there exists $\varsigma>0$ such that for every solution $\left(z_{\mathrm{ex}}^{\varsigma}, m_{\mathrm{ex}}^{\varsigma}\right)$ from $\left(\mathcal{X}_{\xi} \cap S^{\star}\right) \times \Omega$ to an outer perturbation ${ }^{5}$ of $\mathcal{H}_{\text {ex }}$ there exists a solution $\left(z_{\text {ex }}, m_{\text {ex }}\right)$ to $\mathcal{H}_{\text {ex }}$ from $\left(\mathcal{X}_{\xi} \cap S^{\star}\right) \times \Omega$ such that $\left(z_{\mathrm{ex}}^{\varsigma}, m_{\mathrm{ex}}^{\varsigma}\right)$ and $\left(z_{\mathrm{ex}}, m_{\mathrm{ex}}\right)$ are $\left(\tau^{\prime}, \varepsilon\right)$-close. Since the mismatch between $\widetilde{f}$ and $f$ in (16) and the mismatch between $\widetilde{g}$ and $g$ in (17) are upper bounded by $\rho \max \{|\Delta A|,|\Delta H|\} \max _{x \in \mathcal{R}_{\tau^{\prime}}^{x}(\mathcal{K})}|x|$, we have that if

\footnotetext{
$\overline{5}$ See [17, Definition 6.27].
}

$|\Delta A|$ and $|\Delta H|$ are such that

$$
\max \{|\Delta A|,|\Delta H|\} \max _{x \in \mathcal{R}_{\tau^{\prime}}^{x}(\mathcal{K})}|x| \leq \frac{\varsigma}{\rho},
$$

the claim follows by relating the solutions of $\mathcal{H}_{\mathrm{ex}}$ to solutions of $\mathcal{H}$, and the solutions of the outer perturbation of $\mathcal{H}_{\text {ex }}$ to solutions of $\widetilde{\mathcal{H}}$.

In simple terms, the above result establishes that the solutions resulting with $\Delta A$ and $\Delta H$ small enough do not differ much from those without such unmodeled dynamics. In particular, for piecewise constant measurement noise as in Theorem 3.3 and $\tau^{\prime}>\delta$, the recurrent finite time convergence property in Theorem 3.3 is practically preserved up to $t=\tau^{\prime}$.

Remark 3.8 In general, though not pursued here, the result in Theorem 3.7 is also applicable to the case when the measurement noise has a specific statistical property, such as Gaussian noise. See the example in Section 4.2 for a discussion about Gaussian noise.

\section{Simulation Results}

\subsection{Piecewise-constant noise with unmodeled dynamics}

For the plant in (1) with $A=\left[\begin{array}{rr}0 & 1 \\ -1 & 0\end{array}\right], H=\left[\begin{array}{ll}1 & 2 \\ 2 & 1\end{array}\right]$,

and parameters $\delta=1, \quad L_{1}=\left[\begin{array}{rr}-1.67 & 3.33 \\ 3.33 & -1.67\end{array}\right], L_{2}=$ $\left[\begin{array}{rr}-2.67 & 5.33 \\ 5.33 & -2.67\end{array}\right]$, then the invertibility of $\Upsilon$ can be verified as $\operatorname{det}(\Upsilon) \approx 0.01$. Moreover, $L_{1}$ and $L_{2}$ are such that $F_{1}$ and $F_{2}$ are Hurwitz. Hence, Assumption 1.1 holds. We perform simulations of the interconnection between the observer $\mathcal{H}_{o}$ and the plant with measurement noise $m(t)=\tilde{c}_{1} \chi_{B_{1}}(t)+\tilde{c}_{2} \chi_{B_{2}}(t)+\tilde{c}_{3} \chi_{B_{3}}(t)$ with $\tilde{c}_{1}=$ $(0.3,0.2), \tilde{c}_{2}=(0.4,0.4), \tilde{c}_{3}=(0.2,0.3), B_{1}=[0,2.5)$, $B_{2}=[2.5,4.5)$, and $B_{3}=[4.5, \infty)$, and from initial conditions $x(0,0)=(0.3,0.4), \hat{x}_{1}(0,0)=\hat{x}_{2}(0,0)=\hat{x}_{0}(0,0)=$ $0, \xi_{1}(0,0)=\xi_{2}(0,0)=0$, and $\hat{m}(0,0)=0$. Figures $2(\mathrm{a})$ and $2(\mathrm{~b})$ show the estimation errors of the proposed observer (solid) and measurement noise with no unmodeled dynamics as well as those of a Luenberger observer with $L_{0}=L_{2}$ (dashed). Since the initial condition is in the set $S$, after the jump at $t=1$ the estimation error of the proposed observer for each state component is zero. This illustrates Theorem 3.1. The noise changes at $t=2.5$, which generates an increase of the error immediately. But once $t=4$ (within $3 \delta$ after the first jump), the estimation error is again zero. This illustrates Theorem 3.3 , indicating that $\tilde{I}_{1}=[1,2.5)$. 

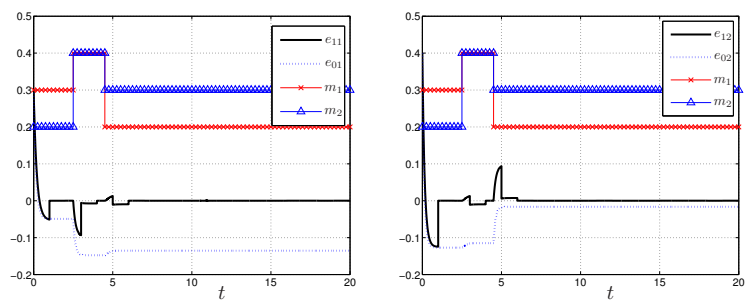

(a) Estimation error for (b) Estimation error for first state component second state component $(\Delta A=0$ and $\Delta H=0)$.

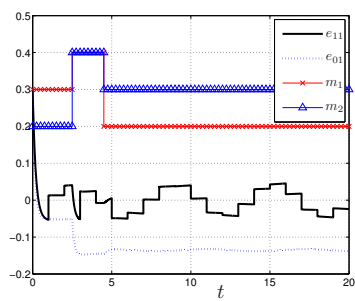

$\Delta A=0$ and $\Delta H=0)$

(c) Estimation error for nonzero unmodeled dy- with nonzero unmodeled namics $\left(\Delta A=\Delta A^{\star}\right.$ and dynamics $\left(\Delta A=\Delta A^{\star}\right.$ and $\left.\left.\Delta H=\Delta H^{\star}\right) . \quad \Delta H=\Delta H^{\star}\right)$.

Fig. 2. State estimates $\left(\hat{x}_{1}=\left(\hat{x}_{11}, \hat{x}_{12}\right)\right)$, measurement noise $\left(m=\left(m_{1}, m_{2}\right)\right)$ and estimation error of the proposed observer $\left(e_{1}=\left(e_{11}, e_{12}\right)=\hat{x}_{1}-x\right)$. $(a)$ and $(b)$ show the situation without unmodeled dynamics, while $(c)$ and $d$ are with unmodeled dynamics.

Figures 2(c) and 2(d) show the estimation errors and measurement noise with unmodeled dynamics $\Delta A=$ $\Delta A^{\star}:=\left[\begin{array}{rr}0.01 & 0.01 \\ 0.01 & 0\end{array}\right], \Delta H=\Delta H^{\star}:=\left[\begin{array}{ll}0.002 & 0.015 \\ 0.011 & 0.003\end{array}\right]$. Although the finite-time convergence property is no longer preserved, the performance of the proposed observer is better than that of the Luenberger observer with $L_{0}=$ $L_{2}$.

\subsection{General noise with recursive jumps}

Consider the same plant as defined in Section 4.1 with the hybrid observer $\mathcal{H}_{o}$ under the general measurement noise $m(t)=\tilde{c}_{1} \chi_{B_{1}}(t)+\tilde{c}_{2} \chi_{B_{2}}(t)+\tilde{c}_{3} \chi_{B_{3}}(t)+$ $0.05[\sin (t) \cos (t)]^{\top}$. Using the same gains $L_{1}, L_{2}$, $L_{0}=L_{2}$, and $\delta=1$ as in Section 4.1, a simulation result is shown in Figure 3 with initial conditions $x(0,0)=(0.3,0.4), \hat{x}_{1}(0,0)=\hat{x}_{2}(0,0)=\hat{x}_{0}(0,0)=0$, $\xi_{1}(0,0)=\xi_{2}(0,0)=0$, and $\hat{m}(0,0)=0$. In Figure 3(a) and $3(\mathrm{~b})$, black solid lines denote the estimation errors of the states from the proposed observer, blue dot-dashed lines denote estimate errors resulting from the Luenberger observer. As the figure shows, the proposed observer provides better estimates. This is also confirmed from Figure 3(c) and 3(d), where nonzero unmodeled dynamics are considered. Estimation errors of the proposed observer are closer to zero comparing to that of a Luenberger observer. Moreover, for the case of Gaussian noise, Table 1 suggests significant improvement on the mean value of the estimation error of the finite-time convergent observer over that of the Luenberger observer.
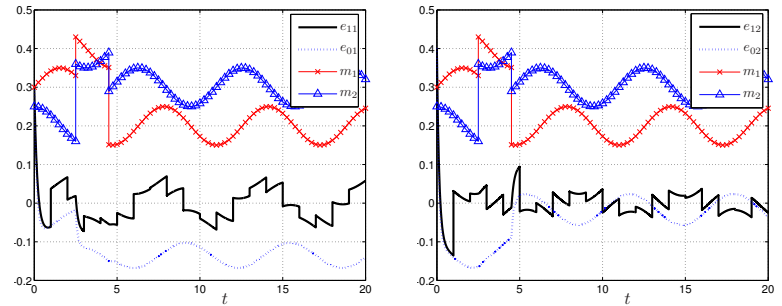

(a) Estimation error for (b) Estimation error for first state component second state component $(\Delta A=0$ and $\Delta H=0)$.

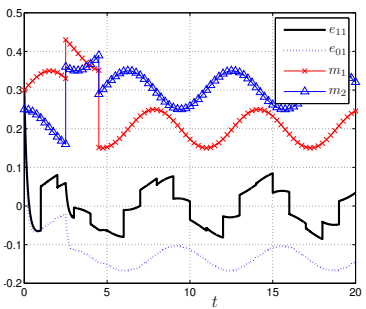

$\Delta A=0$ and $\Delta H=0)$

(c) Estimation error for (d) Estimation error for first state component with second state component nonzero unmodeled dynam- with nonzero unmodeled ics $\left(\Delta A=\Delta A^{\star}\right.$ and $\Delta H=$ dynamics $\left(\Delta A=\Delta A^{\star}\right.$ and $\left.\Delta H^{\star}\right)$.

$\left.\Delta H=\Delta H^{\star}\right)$

Fig. 3. State estimates $\left(\hat{x}_{1}=\left(\hat{x}_{11}, \hat{x}_{12}\right)\right)$, measurement noise $\left(m=\left(m_{1}, m_{2}\right)\right)$ and estimation error $\left(e_{1}=\left(e_{11}, e_{12}\right)=\hat{x}_{1}-x\right) .(a)$ and $(b)$ show the situation without unmodeled dynamics, while $(c)$ and $d$ are with unmodeled dynamics.

Table 1

Comparison of estimation error $\left(e_{i}\right)$ of the observers with zero-mean Gaussian measurement noise (standard deviation is 0.08 )

\begin{tabular}{|l|c|c|}
\hline \multirow{2}{*}{ observer type } & \multicolumn{2}{|c|}{ Estimation error } \\
\cline { 2 - 3 } & mean & std \\
\hline Luenberger & $(-0.139,-0.015)$ & $(0.023,0.024)$ \\
\hline Finite-time & $(-0.002,-0.003)$ & $(0.051,0.039)$ \\
\hline
\end{tabular}

\section{Conclusion}

In this work, a robust finite-time hybrid observer is proposed. When the measurement noise is an unknown piecewise-constant signal (time-varying bias, coarsely quantized noise, etc.), finite-time convergence is guaranteed. Furthermore, for generic measurement noise, the estimation error satisfies $\mathcal{K} \mathcal{L}$-like bounds. Numerical results confirm the results concerning to finite-time convergence and robustness. 


\section{Acknowledgements}

This research has been partially supported by the National Science Foundation under CAREER Grant no. ECS-1150306 and by the Air Force Office of Scientific Research under Grant no. FA9550-12-1-0366.

\section{References}

[1] L. K. Vasiljevic and H. K. Khalil. Differentiation with HighGain Observers the Presence of Measurement Noise. In 45th IEEE Conference on Decision and Control, pages 4717-4722, Dec. 2006.

[2] A. A. Ball and H. K. Khalil. High-gain observers in the presence of measurement noise: A nonlinear gain approach. In 47 th IEEE Conference on Decision and Control, pages 2288-2293, Dec. 2008.

[3] J. H. Ahrens and H. K. Khalil. High-gain observers in the presence of measurement noise: A switched-gain approach. Automatica, 45(4):936-943, 2009.

[4] A. A. Ball and H. K. Khalil. Analysis of a nonlinear high-gain observer in the presence of measurement noise. In Proc. of American Control Conference, pages 2584-2589, July 2011.

[5] R. G. Sanfelice and L. Praly. On the performance of high-gain observers with gain adaptation under measurement noise. Automatica, 47(10):2165-2176, 2011.

[6] H. Lei, J. Wei, and W. Lin. A global observer for observable autonomous systems with bounded solution trajectories. In Proc. of 44th IEEE Conference on Decision and Control, pages 1911-1916, Dec. 2005.

[7] Yuchun Li and R. G. Sanfelice. Robust distributed state observers with performance guarantees and optimized communication graph. In American Control Conference, pages 1090-1095, June 2014.

[8] T. Raff and F. Allgower. An impulsive observer that estimates the exact state of a linear continuous-time system in predetermined finite time. In Proc. Mediterranean Conf. Control and Automation, pages 1-3, 2007.

[9] R. Engel and G. Kreisselmeier. A continuous-time observer which converges in finite time. IEEE Transactions on Automatic Control, 47(7):1202-1204, 2002.

[10] T. Raff, P. Menold, C. Ebenbauer, and F. Allgower. A finite time functional observer for linear systems. In Proc. of 44 th IEEE Conference on Decision and Control, pages 7198-7203, Dec. 2005.

[11] T. Raff, F. Lachner, and F. Allgower. A finite time unknown input observer for linear systems. In Proc. 14th Mediterranean Conference on Control and Automation, pages $1-5$, June 2006.

[12] T. Raff and F. Allgower. An observer that converges in finite time due to measurement-based state updates. In IFAC World Congress, Seoul, Korea, 2008.

[13] W. Perruquetti, T. Floquet, and E. Moulay. Finite-time observers: Application to secure communication. IEEE Transactions on Automatic Control, 53(1):356-360, 2008.

[14] Y. Shen and X. Xia. Semi-global finite-time observers for nonlinear systems. Automatica, 44(12):3152-3156, 2008.

[15] T. Menard, E. Moulay, and W. Perruquetti. A global highgain finite-time observer. IEEE Transactions on Automatic Control, 55(6):1500-1506, 2010.
[16] Y. Shen, Y. Huang, and J. Gu. Global finite-time observers for Lipschitz nonlinear systems. IEEE Transactions on Automatic Control, 56(2):418-424, 2011.

[17] R. Goebel, R. G. Sanfelice, and A. Teel. Hybrid Dynamical Systems: Modeling, Stability, and Robustness. Princeton University Press, 2012.

[18] J. Thienel and R.M. Sanner. A coupled nonlinear spacecraft attitude controller and observer with an unknown constant gyro bias and gyro noise. IEEE Transactions on Automatic Control, 48(11):2011-2015, Nov 2003.

[19] R. Goebel, R. G. Sanfelice, and A. Teel. Hybrid dynamical systems. IEEE Control Systems Magazine, 29(2):28-93, April 2009.

[20] R. G. Sanfelice. Input-output-to-state stability tools for hybrid systems and their interconnections. IEEE Transactions on Automatic Control, 59(5):1360-1366, May 2014.

[21] M. J. Corless and A. E. Frazho. Linear Systems and Control: An Operator Perspective. New York: Marcel Dekker, 2003.

[22] C. Moler and C. Van Loan. Nineteen dubious ways to compute the exponential of a matrix, twenty-five years later. SIAM Review, 45(1):3-49, 2003.

[23] K. B. Petersen and M. S. Pedersen. The Matrix Cookbook, 2006.

[24] Y. Li and R. G. Sanfelice. A robust finite-time convergent hybrid observer for linear systems. In Proc. of 52nd IEEE Conference on Decision and Control, pages 3349-3354, Dec. 2013.

\section{A Finite-time Convergence with Constant Measurement Noise}

The following result is used in the proof of Theorem 3.3

Lemma A.1 For the interconnection between $\mathcal{H}_{o}$ and the plant (1), assume the noise $m:[0, \bar{T}) \rightarrow \mathbb{R}^{p}$ is constant where $\bar{T} \in(\delta, \infty]$. Moreover, suppose Assumption 1.1 holds and $\Upsilon$ is invertible. Then, for any initial condition $z(0,0) \in S^{\star \star}$ with $S^{\star \star}=\left\{z \in \mathbb{R}^{n} \times \mathcal{X}_{o}: \hat{x}_{1}=\right.$ $\left.\hat{x}_{2}, \xi_{1}=\xi_{2}=0, \tau=0\right\}$, the states $\hat{x}_{1}$ and $\hat{x}_{2}$ converge to $x$ within finite time $\delta$, i.e., $\hat{x}_{1}(t, j)=\hat{x}_{2}(t, j)=x(t, j)$ for all $(t, j) \in\{(t, j) \in \operatorname{dom} z: t \in[\delta, \bar{T}), j \geq 1\}$.

Proof From the initial condition $z(0,0) \in S^{\star \star}$, by definition of the flow map, we have

$e_{i}(t, 0)=\exp \left(F_{i} t\right) e_{i}(0,0)+\int_{0}^{t} \exp \left(F_{i}(t-\tau)\right) L_{i}(m-\hat{m}) d \tau$

and $\xi_{i}(t, 0)=\int_{0}^{t} \operatorname{diag}_{p}\left(\exp \left(\left(A-L_{i} H\right)(t-\tau)\right)\right) \tilde{L}_{i} d \tau$ for all $(t, j) \in[0, \delta] \times\{0\}$. When $t=\delta$ and $j=1$, using the definitions of $K_{1}$ and $K_{2}$, and the fact that $\hat{x}_{i}=e_{i}+x$ and $m$ and $\hat{m}$ are constant over $[0, \delta]$, we get

$$
\begin{aligned}
& \hat{m}(\delta, 1)=\hat{m}(\delta, 0) \\
& \quad+(H T(\delta, 0)-I)^{-1}(H R(\delta, 0)-(y(\delta, 0)-\hat{m}(\delta, 0))) \\
& =m(\delta, 0),
\end{aligned}
$$


which implies that $\hat{x}_{1}(\delta, 1)=\hat{x}_{2}(\delta, 1)=x(\delta, 1)$. Since $x(\delta, 1)=x(\delta, 0)$, we have finite time convergence of $e_{i}$ 's to zero.

\section{B A Bound on the Estimation Error when Using (4) under the Presence of Measurement Noise}

The observer (4) can be rewritten as a hybrid system, denoted by $\mathcal{H}_{n}$, given by

$$
\begin{aligned}
\dot{\zeta} & =f_{n}(\zeta, y) \quad \zeta \in C_{n}, \\
\zeta^{+} & =g_{n}(\zeta, y) \quad \zeta \in D_{n},
\end{aligned}
$$

where $\zeta=\left(\hat{x}_{1}, \hat{x}_{2}, \tau, q\right) \in \mathcal{X}_{0}:=\mathbb{R}^{2 n} \times[0, \delta] \times\{0,1\}$. The flow set is defined as $C_{n}=\mathcal{X}_{0}$ and the jump set is $D_{n}=\left\{\zeta \in \mathcal{X}_{0}: \tau=\delta, q=0\right\}$. While the flow map and jump map are given by

$f_{n}(\zeta, y)=\left[\begin{array}{l}F_{1} \hat{x}_{1}+L_{1} H x \\ F_{2} \hat{x}_{2}+L_{2} H x \\ 1-q \\ 0\end{array}\right], g_{n}(\zeta, y)=\left[\begin{array}{l}K_{1} \hat{x}_{1}+K_{2} \hat{x}_{2} \\ K_{1} \hat{x}_{1}+K_{2} \hat{x}_{2} \\ 0 \\ 1-q\end{array}\right]$,

where $K_{1}=I-K_{2}$ and $K_{2}=\left(I-\exp \left(F_{2} \delta\right) \exp \left(-F_{1} \delta\right)\right)^{-1}$. For the case where the noise $m$ is a general function, the interconnection between $\mathcal{H}_{n}$ and the plant is given by

$$
\begin{array}{ll}
\dot{z}_{m}=\left(A x, f_{n}(\zeta, y)\right)=: f_{m}\left(z_{m}\right) & z_{m} \in C_{m}, \\
z_{m}^{+}=(x, g(\zeta))=: g_{m}\left(z_{m}\right) & z_{m} \in D_{m},
\end{array}
$$

where $z_{m}=(x, \zeta) \in \mathbb{R}^{n} \times \mathcal{X}_{0}$, and $y=H x+m$. Moreover, $C_{m}:=\mathbb{R}^{n} \times C_{n}$ and $D_{m}:=\mathbb{R}^{n} \times D_{n}$. Denote the hybrid system in (B.3) by $\mathcal{H}_{m}$. (For a concept of a solution to this system under the presence of measurement noise, see [17, Definition 4.6].)

We derive a bound on the estimation error. For (B.3), the estimation error after the jump can be calculated as

$e_{1}(\delta, 1)=e_{2}(\delta, 1)=\sum_{i=1}^{2} K_{i} \int_{0}^{\delta} \exp \left(F_{i}(\delta-\tau)\right) L_{i} m(\tau) d \tau$.

Then, the following bound can be established.

Proposition B.1 For the hybrid system (B.3), suppose $\bar{\alpha}\left(F_{2}\right)<\underline{\alpha}\left(F_{1}\right)$ and $\bar{\alpha}\left(F_{1}\right)<0$. If $F_{1}$ and $F_{2}$ are dissipative and $\overline{\bar{\mu}}\left(F_{2}\right)<\mu\left(F_{1}\right)$, then (B.4) satisfies

$\left|e_{1}(\delta, 1)\right| \leq\left(\left(1+\bar{w}_{1}(\delta)\right) \frac{\left|L_{1}\right|}{\left|\bar{\mu}\left(F_{1}\right)\right|}+\bar{w}_{1}(\delta) \frac{\left|L_{2}\right|}{\left|\bar{\mu}\left(F_{2}\right)\right|}\right)|m|_{\infty}$,

where $\bar{w}_{1}(\delta)=\frac{1}{1-\exp \left(\left(\bar{\mu}\left(F_{2}\right)-\underline{\mu}\left(F_{1}\right)\right) \delta\right)}$.
Proof By using $\log$ norms [22], $\exp \left(F_{i} \delta\right)$ can be bounded as $\left|\exp \left(F_{i} \delta\right)\right| \leq \exp \left(\bar{\mu}\left(F_{i}\right) \delta\right)$ and $\left|\exp \left(-F_{i} \delta\right)\right| \leq$ $\exp \left(-\mu\left(F_{i}\right) \delta\right)$, for $i=1,2$ and $\delta>0$. With the assumption that $\bar{\mu}\left(F_{2}\right)<\mu\left(F_{1}\right)$, for any $\delta>0$,

$\left|\exp \left(F_{2} \delta\right) \exp \left(-F_{1} \delta\right)\right| \leq \exp \left(\left(\bar{\mu}\left(F_{2}\right)-\underline{\mu}\left(F_{1}\right)\right) \delta\right)<1$.

Then, it follows that, for any fixed $\delta>0$,

$$
\lim _{q \rightarrow \infty}\left(\exp \left(F_{2} \delta\right) \exp \left(-F_{1} \delta\right)\right)^{q}=0
$$

[23]. On the other hand, as $F_{1}$ and $F_{2}$ are dissipative, we have, for $i \in\{1,2\}$

$$
\left|\int_{0}^{\delta} \exp \left(F_{i}(\delta-\tau)\right) L_{i} m(\tau) d \tau\right| \leq \frac{\left|L_{i}\right|}{\left|\bar{\mu}\left(F_{i}\right)\right|}|m|_{\infty}
$$

By applying Taylor expansion [23] and using (B.6), it follows that, for $\delta>0$,

$$
\left|K_{2}\right| \leq 1+\exp (\Delta F \delta)+(\exp (\Delta F \delta))^{2}+\cdots,
$$

where $\Delta F:=\bar{\mu}\left(F_{2}\right)-\mu\left(F_{1}\right)$. Since $\exp (\Delta F \delta)<1$, the series is convergent and we have

$$
\left|K_{2}\right| \leq \frac{1}{1-\exp (\Delta F \delta)} .
$$

Then, by using the fact $K_{1}=I-K_{2}$, it follows that

$$
\left|K_{1}\right| \leq 1+\frac{1}{1-\exp (\Delta F \delta)}
$$

Therefore, by using (B.7), (B.8) and (B.9), (B.4) can be bounded as

$$
\begin{aligned}
\left|e_{1}^{+}(\delta)\right|= & \left|e_{2}^{+}(\delta)\right| \leq\left(1+\frac{1}{1-\exp (\Delta F \delta)}\right) \frac{\left|L_{1}\right|}{\left|\bar{\mu}\left(F_{1}\right)\right|}|m|_{\infty} \\
& +\frac{1}{1-\exp (\Delta F \delta)} \frac{\left|L_{2}\right|}{\left|\bar{\mu}\left(F_{2}\right)\right|}|m|_{\infty} .
\end{aligned}
$$

Remark B.2 Define the function $\delta \mapsto \Gamma(\delta)$ by

$$
\Gamma(\delta)=\left(1+\bar{w}_{1}(\delta)\right) \frac{\left|L_{1}\right|}{\left|\bar{\mu}\left(F_{1}\right)\right|}|m|_{\infty}+\bar{w}_{1}(\delta) \frac{\left|L_{2}\right|}{\left|\bar{\mu}\left(F_{2}\right)\right|}|m|_{\infty},
$$

then $\delta \rightarrow \Gamma(\delta)$ is a strictly decreasing function on $(0, \infty)$. Furthermore, $\lim _{\delta \rightarrow 0} \Gamma(\delta)=\infty$ and $\lim _{\delta \rightarrow \infty} \Gamma(\delta)=\frac{2\left|L_{1}\right|}{\left|\bar{\mu}\left(F_{1}\right)\right|}|m|_{\infty}+\frac{\left|L_{2}\right|}{\left|\bar{\mu}\left(F_{2}\right)\right|}|m|_{\infty}$.

Based on (B.5), a $\mathcal{K} \mathcal{L}$ bound can be derived for $e_{i}$ 's for all $(t, j) \in \operatorname{dom} e_{i}$, see [24]. 